\title{
Uma comparação entre incisões oblíquas e verticais na coleta do tendão dos isquiotibiais na reconstrução do LCA e na lesão do ramo infrapatelar do nervo safeno*
}

\section{A Comparison between Oblique and Vertical Incisions on the Hamstring Tendon Harvesting in Anterior Cruciate Ligament Reconstruction and Infrapatellar Branch Injury of the Saphenous Nerve}

Sohrab Keyhani ${ }^{1}$ Seyyed Morteza Kazemi ${ }^{1} \quad$ Mohammadreza Minator Sajjadi $^{2} \quad$ Asghar Elmi $^{1,30}$

\footnotetext{
1 Departamento Ortopédico, Shahid Beheshti University of Medical Sciences, Akhtar Hospital, Tehran, Iran

2 Departamento Ortopédico, Shahid Beheshti University of Medical Sciences, Taleghani Hospital, Tehran, Iran

${ }^{3}$ Departamento Ortopédico, Tabriz University of Medical Sciences, Shohada Hospital, Tabriz, Iran
}

\begin{abstract}
Endereço para correspondência Asghar Elmi, MD, Knee Fellowship Surgery, Orthopedic Department, Tabriz University of Medical Science, Shohada Hospital, Tabriz, Iran (e-mail: elmimail@yahoo.com).
\end{abstract}

Rev Bras Ortop 2020;55(3):374-379.

\begin{abstract}
Resumo
Palavras-chave

- ligamento cruzado anterior

- incisão oblíqua

- reconstrução

- nervo safeno

Objetivo $\mathrm{O}$ presente estudo teve como objetivo comparar as incisões oblíquas e verticais na coleta dos tendões dos isquiotibiais na reconstrução do ligamento cruzado anterior (LCA) e na lesão do ramo infrapatelar do nervo safeno.

Métodos O presente estudo foi realizado em um centro de referência terciário por 12 meses. Pacientes com indicação de reconstrução de uma ruptura do LCA foram incluídos no estudo, os quais foram randomizados em dois grupos (vertical [GV] e oblíquo [GO]). Após a exclusão de alguns casos, 92 pacientes foram elegíveis para análise posterior (GV: $n=44$; GO: $n=48$ ). Eles foram acompanhados por 9 meses após a cirurgia, e a perda de sensibilidade sobre o joelho e sobre o aspecto proximal da perna operada foi registrado.

Resultados Os comprimentos médios da incisão foram de $27 \mathrm{~mm}$ e $38 \mathrm{~mm}$ para os grupos $\mathrm{GO}$ e GV, respectivamente. A taxa total de hipoestesia foi de $40 \%$ (27 pacientes). Um total de 12 (25\%) e de 25 pacientes (56,8\%) dos grupos GO e GV, respectivamente, relataram sintomas de hipoestesia. A presença de hipoestesia em pacientes no grupo GV foi duas vezes maior do que no grupo GO. Não foi observada correlação estatística entre a lesão do nervo e idade, gênero, escolaridade e demora entre a lesão e a reconstrução.
\end{abstract}

\footnotetext{
Trabalho desenvolvido na Universidade Shahid Beheshti de Ciências Médicas, Hospital Akhtar, Teerã, Irã
}

recebido

21 de Março de 2019

aceito

13 de Maio de 2019
DOI https://doi.org/

10.1055/s-0039-1692695. ISSN 0102-3616.
Copyright $\odot 2020$ by Sociedade Brasileira License terms de Ortopedia e Traumatologia. Published by Thieme Revnter Publicações Ltda, Rio de Janeiro, Brazil 


\begin{abstract}
Keywords

- anterior cruciate ligament

- oblique incision

- reconstruction

- saphenous nerve

Objective The present study aimed to compare the oblique and vertical incisions in hamstring tendon harvesting in anterior cruciate ligament (ACL) reconstruction and in infrapatellar branch injury of the saphenous nerve.

Methods The present study was conducted at a tertiary referral center for 12 months. Patients with an indication of reconstruction of $A C L$ tear were included in the study, who were then randomized into two groups (vertical [VG] and oblique [OG] groups). After excluding a few cases, 92 patients were eligible for further analysis (VG: $n=44$; OG: $n=48$ ). They were followed-up for 9 months after the surgery, and loss of sensation over the knee and over the proximal aspect of the operated leg was recorded. Results The mean lengths of the incisions were $27 \mathrm{~mm}$ and $38 \mathrm{~mm}$ for the OG and VG groups, respectively. The total rate of hypoesthesia was $40 \%$ ( 27 patients). A total of 12 (25\%) and 25 patients (56.8\%) on the OG and VG groups, respectively, reported hypoesthesia symptoms. The presence of hypoesthesia in patients in the VG group was two times higher than in the OG group. No statistical correlation was observed between the nerve injury and age, gender, education, and delay from injury to reconstruction.

Conclusion Oblique incision, which showed lower risk of nerve damage, might be more recommended for graft harvesting. Patients who underwent reconstruction of the $A C L$ in the $O G$ group had a lower incidence of peri-incisional hypoesthesia when compared to those in the VG group.
\end{abstract}

Conclusão A incisão oblíqua, que apresentou menor risco de lesão nervosa, pode ser mais recomendada para a coleta do enxerto. Pacientes submetidos à reconstrução do LCA no grupo GO tiveram menor incidência de hipoestesia peri-incisional quando comparados aos pacientes do grupo GV.

\section{Introdução}

O ligamento cruzado anterior (LCA) é um dos principais ligamentos lesados comumente no joelho. Estima-se que entre 200 mil lesões do LCA a cada ano nos Estados Unidos, quase a metade delas seja submetida a reconstrução do LCA. ${ }^{1}$ Independentemente disso, as escolhas e técnicas de enxerto usadas para colhê-los são imperativas. As opções de enxerto autólogo mais utilizadas incorporam o terço central do tendão patelar, do quadríceps, e dos tendões isquiotibiais (semitendíneo e grácil). ${ }^{2}$ Todos esses métodos têm sido amplamente utilizados, e suas complicações e resultados estão descritos nas publicações. ${ }^{3,4}$

A reconstrução artroscópica do LCA com enxerto dos tendões semitendíneo e grácil requer uma pequena incisão, que é uma opção eficaz e segura, em contraste com o autoenxerto do tendão patelar, com menor morbidade do local doador. ${ }^{4}$ No entanto, devido à localização anatômica específica, existe um risco potencial de dano ao ramo infrapatelar do nervo safeno (RIPNS) durante a retirada do enxerto de isquiotibiais, o que pode causar complicações como disestesia periincisional e dor local. ${ }^{5} \mathrm{~A}$ incidência de qualquer distúrbio sensorial na distribuição do RIPNS decorrente de cirurgia de joelho assistida artroscopicamente, incluindo reconstrução do LCA, tem variado amplamente de estudo para estudo, sendo relatada em até $88 \%$ dos casos. ${ }^{6,7}$
Várias investigações sugerem a utilização de incisões horizontais e oblíquas para expor e colher a inserção tibial dos tendões dos isquiotibiais para reduzir os danos ao RIPNS, em comparação com as incisões verticais; mas não há consenso até o momento. ${ }^{4,8}$ Foi considerado que as incisões com maior respeito à anatomia do RIPNS poderiam garantir uma menor taxa de complicações. Posteriormente, o presente estudo teve como objetivo comparar a incidência de lesão de RIPNS entre duas incisões diferentes, verticais e oblíquas, utilizadas para a coleta do tendão dos isquiotibiais.

\section{Materiais e Métodos}

\section{Pacientes}

A presente pesquisa é um estudo de caso-controle realizado em um centro de referência terciária do Hospital Akhtar, Teerã, Irã, durante 12 meses (de maio de 2017 a abril de 2018). Um total de 245 pacientes foi submetido à reconstrução primária do LCA com enxerto isquiotibial.

O estudo foi realizado em conformidade com a Declaração de Helsinque da Associação Médica Mundial sobre Princípios Éticos para Pesquisa Médica Envolvendo Seres Humanos, e foi revisado pelo Conselho de Revisão Institucional da Universidade de Ciências Médicas Shahid Beheshti. Os autores forneceram consentimento informado por escrito durante o período do estudo para todos os pacientes incluídos. A intervenção foi 
explicada antes da cirurgia para cada paciente, e um questionário foi preenchido para determinar a causa da lesão do LCA, o intervalo da lesão até a cirurgia, idade, gênero e nível de educação. Os pacientes foram randomizados em dois grupos (grupos vertical [GV] e grupo oblíquo [GO]) usando um software de geração de números aleatórios. Números ímpares foram atribuídos ao GV e números pares foram atribuídos ao GO. Todos os pacientes foram submetidos a uma reconstrução primária do LCA com técnica de autoenxerto de isquiotibiais, no mesmo centro de saúde, pela mesma equipe cirúrgica. Lesões meniscais concomitantes foram tratadas por meniscectomia parcial ou reparo usando uma técnica totalmente interna para reparo do menisco. Não houve incisões extras para as cirurgias de menisco.

\section{Critério de Inclusão}

Pacientes com indicação de reconstrução devido a uma ruptura do LCA foram incluídos. Após a cirurgia, 153 pacientes foram excluídos do estudo, devido a cirurgias prévias na região do joelho estudado, a qualquer anormalidade neurológica periférica antes do procedimento, a cicatrizes antigas ao redor do joelho, a lesão multiligamentar, e a perda do acompanhamento. Após a exclusão de todos os casos, 92 pacientes foram elegíveis para análise posterior (GV: $n=44$; GO: $n=48$ ).

\section{Técnica Cirúrgica}

Todas as incisões para a coleta do enxerto foram feitas com o joelho flexionado a $90^{\circ}, 2 \mathrm{~cm}$ abaixo da extremidade superior do tubérculo anterior da tíbia (TAT) e $1 \mathrm{~cm}$ medial ao mesmo. As incisões verticais foram feitas em um plano paralelo ao TAT; as incisões oblíquas foram feitas com uma inclinação de $45^{\circ}$ em relação a este plano, de superomedial a inferolateral (-Fig. 1 e - Fig. 2). ${ }^{9}$ Para colher os tendões, foi feita uma incisão longitudinal de $2 \mathrm{~cm}$. 0 comprimento da incisão foi alongado quando necessário. Os tendões grácil e semitendíneo foram apalpados após a incisão, e a fáscia sartorial sobrejacente foi aberta ao longo da borda superior do tendão grácil. Depois que os dois tendões foram isolados com um grampo, essas estruturas distintas foram destacadas da sua inserção para a tíbia tão distalmente quanto possível, depois foram colhidas com um stripper de tendões de extremidade fechada. A mesma incisão foi então usada para a preparação e colocação do enxerto do LCA. Foi realizada uma reconstrução formal e transportal quádrupla dos isquiotibiais do LCA. Um EndoButton (botão de sutura) foi usado para a fixação femoral, e um parafuso de interferência foi usado para a fixação tibial.

\section{Reabilitação}

O mesmo protocolo de reabilitação foi usado para ambos os grupos. No presente estudo, 9 meses após a cirurgia, os pacientes foram examinados para perda sensorial em torno do joelho por meio do teste de picada de agulha. Em seguida, a área com perda sensorial foi marcada e as fotos digitais foram tiradas com uma régua como escala. Depois, a área de perda sensorial $\left(\mathrm{cm}^{2}\right)$ e o comprimento da incisão $(\mathrm{mm})$ foram medidos pelo software Photoshop (Adobe Inc., Moun-

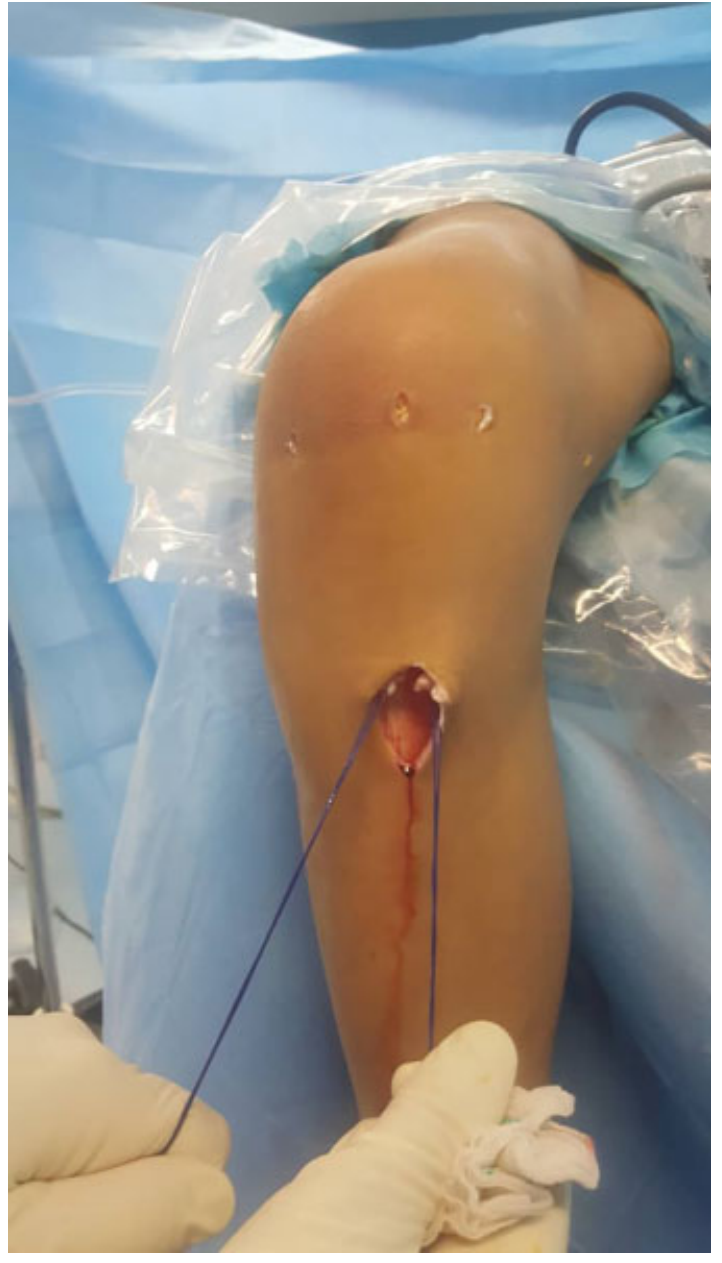

Fig. 1 Incisão vertical para a coleta do tendão dos isquiotibiais.

tain View, CA, EUA). As avaliações clínicas foram realizadas por um único pesquisador.

\section{Análises Estatísticas}

Os dados foram importados para o IBM SPSS Statistics for Windows, Versão 22 (IBM Corp., Armonk, NY, EUA) para análise. Os testes de qui-quadrado e $t$ de Student foram usados para dados não paramétricos e paramétricos, respectivamente. $\mathrm{P}<0,05$ foi considerado estatisticamente significante.

\section{Resultados}

Dentre os 92 pacientes incluídos, 86 eram do sexo masculino e 6 do sexo feminino (idade média $=26,9$ anos, variando de 17 a 47 anos). Um total de 65 (71,6\%) lesões foi devido a lesões esportivas, sendo os acidentes de futebol a causa mais frequente de ruptura do LCA ( $n=45 ; 48,9 \%$ ). Algumas lesões foram causadas por outros esportes que não o futebol ( $n=20 ; 21,7 \%)$, quedas e acidentes com veículos $(n=18$; $19,5 \%$ para ambos), juntamente com algumas outras causas ( $n=9 ; 9,7 \%$ ). Entre os pacientes, 25\% tinham diploma universitário (ou seja, bacharelado, mestrado, doutorado, etc.), $45 \%$ tinham o ensino médio completo, e 30\% tinham 


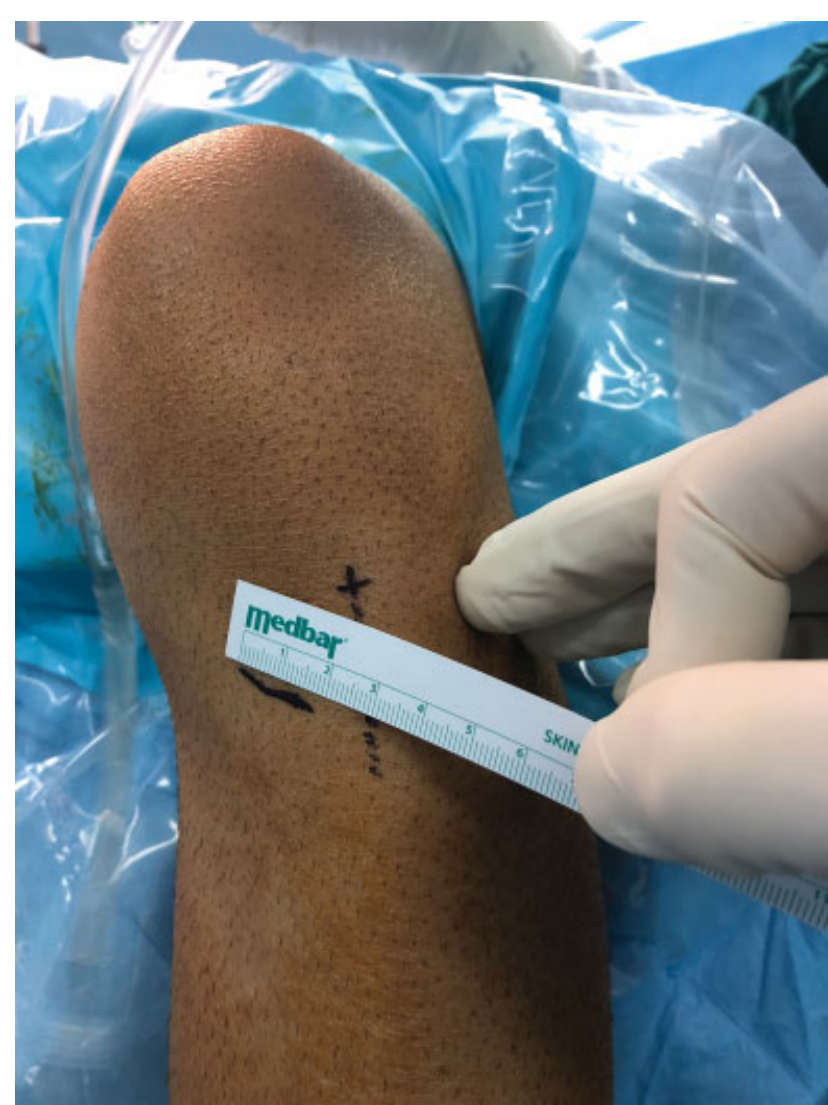

Fig. 2 Incisão oblíqua para a coleta do tendão dos isquiotibiais.

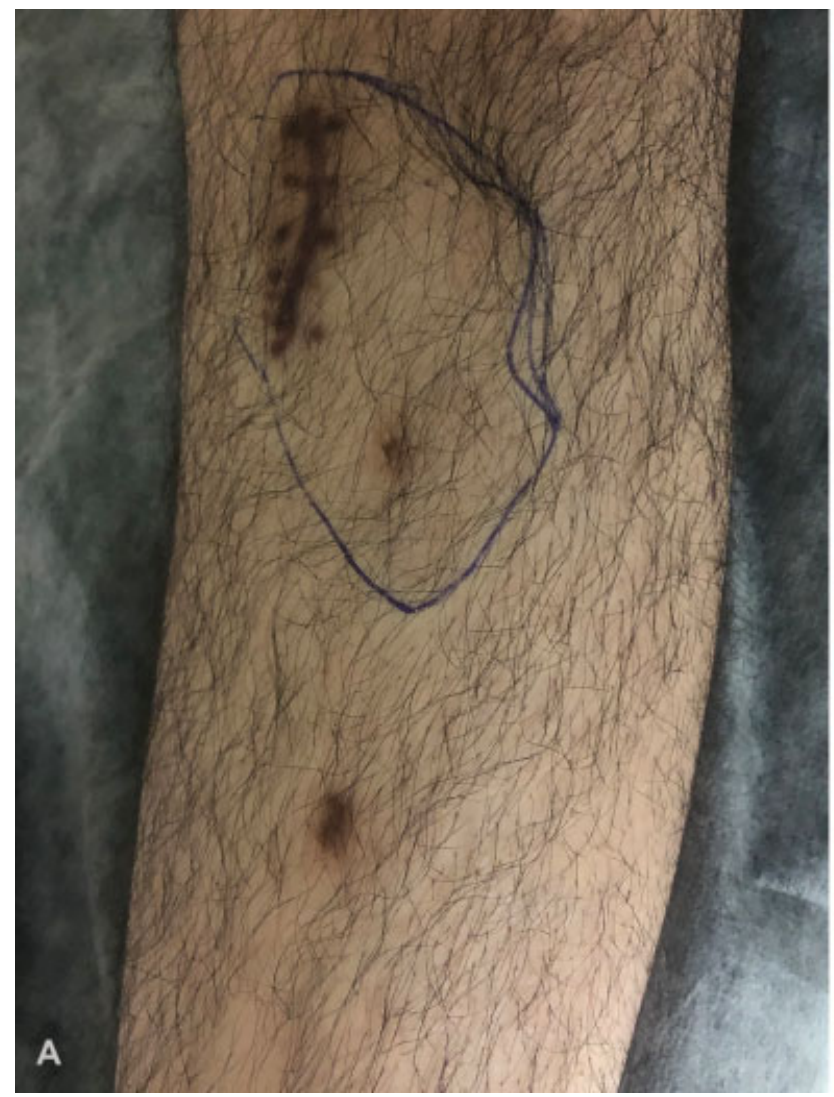

educação inferior ao ensino médio completo. A média de tempo da lesão até a cirurgia foi de 11 meses (com variação de 1,5 a 61 meses), e o comprimento médio da incisão foi de $27 \mathrm{~mm}$ (20 a $39 \mathrm{~mm}$ ) para o GO, e de $38 \mathrm{~mm}$ (25 a $47 \mathrm{~mm}$ ) para o GV. Não houve diferença entre os grupos em relação ao tipo de lesão, bem como não houve diferenças quanto ao tempo de demora para a cirurgia e gênero dos pacientes.

A incisão oblíqua foi utilizada em 48 dos 92 joelhos avaliados. Destes, 12 (25\%) apresentaram hipoestesia em torno do joelho operado e na região proximal da perna após 9 meses de acompanhamento. 0 comprimento médio da hipoestesia no GO foi de $9,6 \mathrm{~cm}^{2}$ (com variação de $1,7 \mathrm{a}$ $20,2 \mathrm{~cm}^{2}$ ) (-Fig. 3). Entre os 44 pacientes com incisão vertical, 25 (56,8\%) apresentaram algum grau de hipoestesia após 9 meses de acompanhamento, com o comprimento médio da hipoestesia sendo de $34,2 \mathrm{~cm}^{2}$ (3,3 a 76,2 $\left.\mathrm{cm}^{2}\right)$ (-Fig. 3). De acordo com o teste de razão de chances, a presença de hipoestesia em pacientes submetidos à incisão vertical foi 2 vezes maior (2,27 vezes; IC [intervalo de confiança] $=95 \%$ ) do que em pacientes operados com acesso oblíquo. Nos pacientes com queixas de hipoestesia, a região anterolateral proximal da perna foi a área mais acometida (75\%). Além disso, o acometimento anteromedial proximal da perna foi observado em $10 \%$ dos pacientes, e o acometimento tanto da região anterolateral quanto da anteromedial foi observado em $15 \%$ dos pacientes. Não houve diferença estatisticamente significativa entre o GO e o GV em relação à localização da hipoestesia.

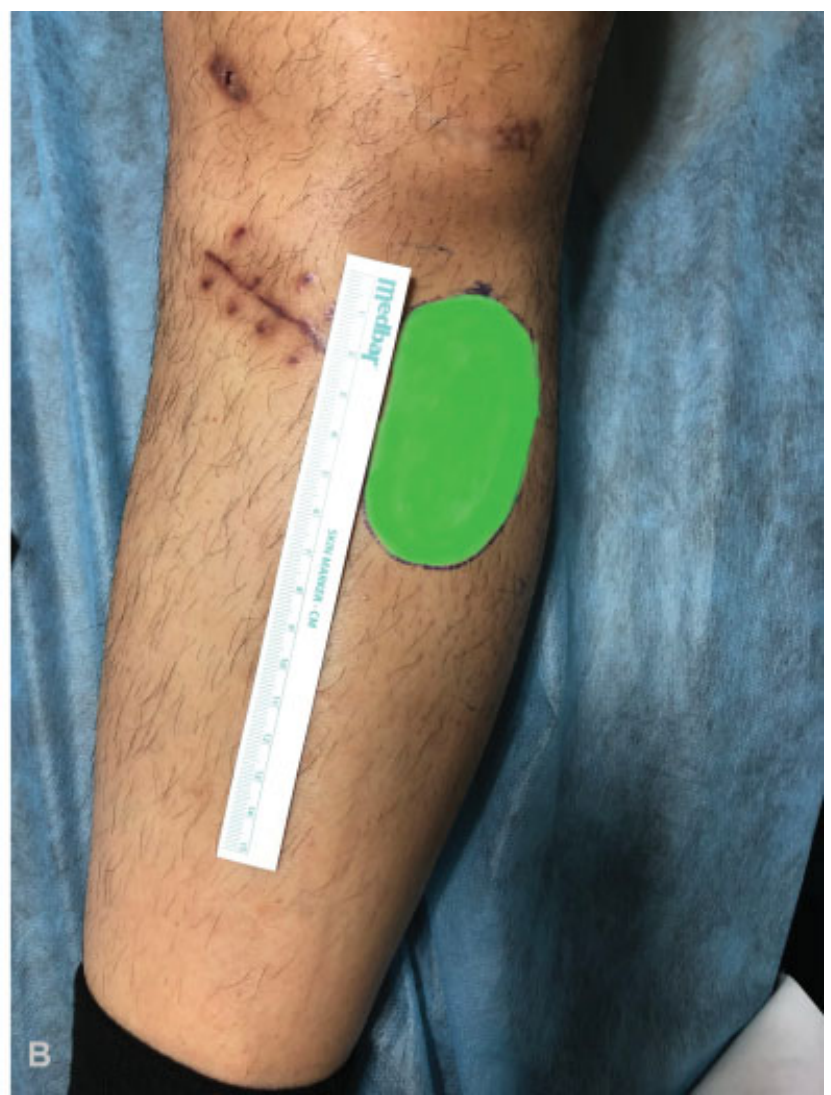

Fig. 3 Área de perturbação sensitiva dos pacientes submetidos à reconstrução do ligamento cruzado anterior, utilizando as incisões vertical (A) e oblíqua (B), e marcação de área. 
Quando os pacientes foram questionados sobre as queixas do RIPNS, a taxa de queixas foi menor do que a razão medida. No entanto, a proporção de queixas subjetivas foi significativamente menor no grupo de incisão oblíqua em comparação com o grupo de incisão vertical $(p<0,05)$. Isso indica que os pacientes não reconheceram este problema ou o negligenciaram. A incidência de qualquer adormecimento relatado para o GO foi de 41,6\% (5/12) (pacientes que relataram dormência/ pacientes com queixas de hipoestesia) aos 9 meses, enquanto a incidência de dormência para o VG foi de 60,0\% (15/25). Não houve diferença estatisticamente significativa entre os grupos comparando o comprimento da incisão e outros dados demográficos. Além disso, a incidência de qualquer queixa relatada sobre dor no local da incisão após 9 meses foi de 6,2\% (3/48) e de 20,4\% (9/44) para o OG e o VG, respectivamente. A dor não interferiu nas atividades diárias de nenhum dos pacientes. Os pacientes que tiveram queixas subjetivas foram vistos como tendo uma área de hipoestesia maior. Idade, gênero, educação e atraso de lesão à reconstrução não mostraram correlação significativa com lesão do nervo e queixas dos pacientes.

\section{Discussão}

No presente estudo, dois tipos de incisões oblíquas e verticais foram comparados sobre a região da "pata de ganso" (pata anserina), através da qual os tendões mediais dos isquiotibiais são colhidos. Além disso, o presente estudo propõe que, em contraste com a incisão vertical tradicional, a incisão oblíqua pode diminuir significativamente a lesão do RIPNS, resultando em pacientes com queixas diminuídas. Uma prevalência global de $40,2 \%$ de hipoestesia foi observada no presente estudo, enquanto uma prevalência duas vezes menor de hipoestesia peri-incisional foi observada no GO quando comparado com o VG, o que pode ser atribuído a uma maior conformidade com a anatomia do RIPNS nesta situação.

Uma incisão padrão não foi descrita na literatura para a coleta de enxerto de semitendíneo e grácil. Brown et al mostraram uma incisão oblíqua em paralelo com as linhas da pele na parte superior da pata anserina. Essa incisão paralela deveria proporcionar uma visão superior e levar a menos cicatrizes, facilitando a retração da pele. ${ }^{10}$ Marder et al $^{11}$ recomendaram uma incisão oblíqua de $4 \mathrm{~cm}, 3$ dedos distais do nível articular sobre a inserção da pata anserina. Em um estudo com cadáveres de Pagnani et al, ${ }^{12}$, eles anunciaram que o local de inserção dos tendões isquiotibiais está a $1,9 \mathrm{~cm}$ distal e $2,25 \mathrm{~cm}$ medial do ápice tubercular da tíbia, e sugeriram a coleta de enxerto de 4 posições do joelho para relaxar o nervo safeno ao passar o tendão grácil no posteromedial do joelho. Considera-se que uma incisão vertical causa mais tecido cicatricial por ser vertical às linhas da pele e também aumenta o risco de lesão do nervo devido a ser vertical ao RIPNS. ${ }^{13}$

O RIPNS sai do canal adutor e passa descendo em um ângulo de $\sim 45^{\circ}$ através do joelho anterior. ${ }^{14}$ Além disso, existem numerosas variedades dos ramos transversais do RIPNS, desde o polo inferior da patela até o tubérculo tibial distal. ${ }^{15}$ Portanto, a lesão nervosa é inescapável quando a pele é incisada para a coleta de tendão. Sabiamente, uma incisão paralela à passagem do nervo pode causar menos lesão do nervo. ${ }^{16}$ Também pode ocorrer lesão do ramo sartorial, o que acabou sendo contabilizado em até $23 \%$ dos pacientes. $^{17}$

Nossos resultados afirmam a teoria de que uma incisão oblíqua pode diminuir os danos nos nervos. Além disso, a dormência estava significativamente ligada à região da perda sensorial e ocorria com menos frequência no OG ( $p<0,001)$. Um exame em joelhos de cadáveres mostrou que o RIPNS é relativamente paralelo à borda superior dos tendões anserinos (medial-superior a lateral-inferior). ${ }^{4} \mathrm{~A}$ proximidade entre essas estruturas, relacionada à posição superficial de seu ramo terminal na região anteromedial do joelho, poderia esclarecer a incidência de lesões iatrogênicas do RIPNS na reconstrução do LCA com tendões flexores, o que, como indicado pela literatura, pode chegar a $77 \% .{ }^{6,18,19}$ Darestani et $\mathrm{al}^{17}$ investigaram 60 casos e descobriram que a incisão oblíqua estava relacionada à diminuição da incidência da lesão; no entanto, essa ligação não foi significativa. A concordância entre especialistas depende justamente da presença de um paralelismo entre as incisões horizontais e oblíquas e a anatomia do RIPNS. Portland et al ${ }^{19}$ analisaram três complicações relacionadas a essas duas incisões na remoção do tendão flexor, incluindo dor, aparência estética e disestesia. Em todos os itens avaliados, a incisão vertical apresentou maior taxa de complicações.

Vários estudos foram realizados para limitar as complicações durante a coleta do enxerto para a reconstrução do LCA. Letartre et $\mathrm{al}^{20}$, para evitar lesões no nervo safeno e em seus ramos na região anteromedial do joelho, sugeriram uma técnica que utiliza o acesso posterior para remoção dos tendões flexores. Em outro exame, de Padua et al $^{21}$ relataram uma menor taxa de lesão do nervo safeno em pacientes em quem apenas o tendão semitendíneo foi removido (preservando assim o grácil) quando comparado com a colheita de ambos os enxertos. Para avaliar o impacto da posição do RIPNS na mobilidade dinâmica do joelho em 20 joelhos de cadáver, Tifford et al $^{13}$ presumiram que as incisões na face anterior do joelho deveriam ser feitas em flexão. Em concordância com esses autores, todos os pacientes em nossa investigação foram submetidos à incisão com o joelho em flexão.

O efeito da lesão do nervo na função do joelho não foi pesquisado, e esta foi a principal limitação do presente exame. Além disso, outros métodos, como estudos eletrofisiológicos, teriam sido mais úteis para a análise do nível de lesão nervosa. Além disso, o efeito funcional nos casos com déficit neurológico não foi determinado.

\section{Conclusão}

Lesões no RIPNS podem ser observadas na coleta do enxerto de isquiotibiais. Entretanto, de acordo com nossos resultados, a incisão oblíqua causa menos lesão ao ramo infrapatelar do nervo safeno na reconstrução artroscópica do LCA com autoenxerto do tendão isquiotibial. Além disso, a perda 
sensorial não prejudica as atividades diárias normais na maioria destes pacientes. Como possível complicação, a lesão nervosa e seu prognóstico benigno devem ser explicados ao paciente antes da cirurgia.

Conflito de Interesses

Os autores declaram não haver conflito de interesses.

\section{Referências}

1 Evans S, Shaginaw J, Bartolozzi A. Acl reconstruction - it's all about timing. Int J Sports Phys Ther 2014;9(02):268-273

2 de Carvalho Júnior LH, Machado SLF, Gonçalves MBJ, et al. Periincisional dysesthesia following anterior cruciate ligament reconstruction using central third of patellar tendon. Rev Bras Ortop 2015;46(01):37-39

3 Carneiro M, Navarro RD, Nakama GY, Barretto JM, Bessa de Queiroz AA, Malheiro Luzo MV. Arthroscopic double-bundle reconstruction of anterior cruciate ligament using hamstring tendon grafts: fixation with two interference screws. Rev Bras Ortop 2015;44(05):441-445

4 Gali JC, Resina AF, Pedro G, et al. Importance of anatomically locating the infrapatellar branch of the saphenous nerve in reconstructing the anterior cruciate ligament using flexor tendons. Rev Bras Ortop 2014;49(06):625-629

5 Temponi E, Carvalho Júnior L, Soares L, Gonçalves M, Leite M, Silva Júnior O. Disestesia peri-incisional após reconstrução do ligamento cruzado anterior: comparação entre dois diferentes tipos de enxerto. Rev Port Ortop Traumatol 2013;21(03): 389-394

6 Figueroa D, Calvo R, Vaisman A, Campero M, Moraga C. Injury to the infrapatellar branch of the saphenous nerve in ACL reconstruction with the hamstrings technique: clinical and electrophysiological study. Knee 2008;15(05):360-363

7 Papastergiou SG, Voulgaropoulos H, Mikalef P, Ziogas E, Pappis G, Giannakopoulos I. Injuries to the infrapatellar branch(es) of the saphenous nerve in anterior cruciate ligament reconstruction with four-strand hamstring tendon autograft: vertical versus horizontal incision for harvest. Knee Surg Sports Traumatol Arthrosc 2006;14(08):789-793

8 Leite ML, Cunha FA, Costa BQ Andrade RM, Diniz Junior JH, Temponi EF. Relationship between peri-incisional dysesthesia and the vertical and oblique incisions on the hamstrings harvest in anterior cruciate ligament reconstruction. Rev Bras Ortop 2016;51(06):667-671
9 Boon JM, Van Wyk MJ, Jordaan D. A safe area and angle for harvesting autogenous tendons for anterior cruciate ligament reconstruction. Surg Radiol Anat 2004;26(03):167-171

10 Brown $\mathrm{CH}$ Jr, Steiner ME, Carson EW. The use of hamstring tendons for anterior cruciate ligament reconstruction. Technique and results. Clin Sports Med 1993;12(04):723-756

11 Marder RA, Raskind JR, Carroll M. Prospective evaluation of arthroscopically assisted anterior cruciate ligament reconstruction. Patellar tendon versus semitendinosus and gracilis tendons. Am J Sports Med 1991;19(05):478-484

12 Pagnani MJ, Warner JJ, O'Brien SJ, Warren RF. Anatomic considerations in harvesting the semitendinosus and gracilis tendons and a technique of harvest. Am J Sports Med 1993;21(04):565-571

13 Tifford CD, Spero L, Luke T, Plancher KD. The relationship of the infrapatellar branches of the saphenous nerve to arthroscopy portals and incisions for anterior cruciate ligament surgery. An anatomic study. Am J Sports Med 2000;28(04):562-567

14 Kalthur SG, Sumalatha S, Nair N, Pandey AK, Sequeria S, Shobha L. Anatomic study of infrapatellar branch of saphenous nerve in male cadavers. Ir J Med Sci 2015;184(01):201-206

15 Hunter LY, Louis DS, Ricciardi JR, O'Connor GA. The saphenous nerve: its course and importance in medial arthrotomy. Am J Sports Med 1979;7(04):227-230

16 Kerver AL, Leliveld MS, den Hartog D, Verhofstad MH, Kleinrensink GJ. The surgical anatomy of the infrapatellar branch of the saphenous nerve in relation to incisions for anteromedial knee surgery. J Bone Joint Surg Am 2013;95(23):2119-2125

17 Tavakoli Darestani R, Bagherian Lemraski MM, Hosseinpour M, Kamrani-Rad A. Electrophysiological assessment of injury to the infra-patellar branch (es) of the saphenous nerve during anterior cruciate ligament reconstruction using medial hamstring autografts: vertical versus oblique harvest site incisions. Arch Trauma Res 2013;2(03):118-123

18 Trescot AM, Brown MN, Karl HW. Infrapatellar saphenous neuralgia - diagnosis and treatment. Pain Physician 2013;16(03):E315-E324

19 Portland GH, Martin D, Keene G, Menz T. Injury to the infrapatellar branch of the saphenous nerve in anterior cruciate ligament reconstruction: comparison of horizontal versus vertical harvest site incisions. Arthroscopy 2005;21(03):281-285

20 Letartre R, Isida R, Pommepuy T, Miletic B. Horizontal posterior hamstring harvest. Orthop Traumatol Surg Res 2014;100(08): 959-961

21 de Padua VB, Nascimento PE, Silva SC, de Gusmão Canuto SM, Zuppi GN, de Carvalho SM. Saphenous nerve injury during harvesting of one or two hamstring tendons for anterior cruciate ligament reconstruction. Rev Bras Ortop 2015;50(05):546-549 